\title{
POLYNOMIAL GROTHENDIECK PROPERTIES
}

\author{
by MANUEL GONZÁLEZ† and JOAQUÍN M. GUTIÉRREZ
}

(Received 3 November, 1993)

\begin{abstract}
A Banach space $E$ has the Grothendieck property if every (linear bounded) operator from $E$ into $c_{0}$ is weakly compact. It is proved that, for an integer $k>1$, every $k$-homogeneous polynomial from $E$ into $c_{0}$ is weakly compact if and only if the space $\mathscr{P}\left({ }^{k} E\right)$ of scalar valued polynomials on $E$ is reflexive. This is equivalent to the symmetric $k$-fold projective tensor product of $E$ (i.e., the predual of $\mathscr{P}\left({ }^{k} E\right)$ ) having the Grothendieck property. The Grothendieck property of the projective tensor product $E \hat{\otimes} F$ is also characterized. Moreover, the Grothendieck property of $E$ is described in terms of sequences of polynomials. Finally, it is shown that if every operator from $E$ into $c_{0}$ is completely continuous, then so is every polynomial between these spaces.
\end{abstract}

1. Introduction. Throughout, $E, F$ will be Banach spaces, and $E^{*}$ the dual of $E$. We denote by $\mathscr{L}(E, F)$ the space of all (linear bounded) operators from $E$ to $F$, and by $\mathscr{G}_{0}(E, F)\left(\mathscr{W}_{G}(E, F)\right)$ the subspace of all (weakly) compact operators. We say that $T \in \mathscr{L}(E, F)$ is completely continuous if it takes weakly convergent sequences into norm convergent sequences, and we write $T \in \mathscr{C} \mathscr{C}(E, F)$.

For an integer $k$, we shall consider the following classes of polynomials:

(a) $\mathscr{P}\left({ }^{k} E, F\right)$ is the space of all $k$-homogeneous (continuous) polynomials from $E$ to $F$;

(b) $\mathscr{P}_{c c}\left({ }^{k} E, F\right)$, the subspace of completely continuous polynomials, i.e., the polynomials taking weakly convergent sequences into norm convergent ones, equivalently, taking weak Cauchy sequences into convergent ones [3, Theorem 2.3];

(c) $\mathscr{P}_{\text {wco }}\left({ }^{k} E, F\right)$, the subspace of weakly compact polynomials;

(d) $\mathscr{P}_{w b}\left({ }^{k} E, F\right)$, the polynomials whose restrictions to bounded subsets of $E$ are weakly continuous; these are compact polynomials. It is well known that $\mathscr{P}_{w b}\left({ }^{k} E, F\right) \subseteq$ $\mathscr{P}_{c c}\left({ }^{k} E, F\right)$, and the equality occurs if and only if $E$ contains no copy of $\ell_{1}$ (see e.g. [14]).

The space of $k$-linear (continuous) mappings from $E^{k}$ to $F$ is denoted by $\mathscr{L}\left({ }^{k} E, F\right)$. To each $P \in \mathscr{P}\left({ }^{k} E, F\right)$ we can associate a unique symmetric $A \in \mathscr{L}\left({ }^{k} E, F\right)$ such that $P(x)=A(x, \ldots, x)$ for all $x \in E$. Whenever $F$ is omitted, it is understood to be the scalar field. For the general theory of polynomials between Banach spaces, we refer to [19].

The projective tensor product of $E$ and $F$ is referred to as $E \hat{\otimes} F$. The closed linear span of the set $\{x \otimes \ldots \otimes x: x \in E\}$ in $\hat{\otimes}^{k} E:=E \hat{\otimes}_{\ldots}^{(k)} \hat{\otimes} E$ is denoted by $\hat{\Delta}^{k} E$. Its dual is isomorphic to $\mathscr{P}\left({ }^{k} E\right)$. The spaces $\mathscr{P}\left({ }^{k} E, F\right)$ and $\mathscr{L}\left(\hat{\Delta}^{k} E, F\right)$ are linearly isomorphic, and the image of $\mathscr{P}_{\text {wco }}\left({ }^{k} E, F\right)$ under this isomorphism is $\mathscr{W}^{r} \mathscr{C}_{0}\left(\hat{\Delta}^{k} E, F\right)$ [22].

We say that $E$ has the Grothendieck property, and write $E \in \mathscr{G}_{R}$, if every sequence in $E^{*}$ converging to zero in the weak-star $\left(w^{*}\right)$ topology, is also weakly null. Equivalently, if every operator $E \rightarrow c_{0}$ is weakly compact.

In this paper, we investigate conditions on $E$ so that every $k$-homogeneous

$\dagger$ Supported in part by DGICYT Grant PB 91-0307 (Spain).

¥ Supported in part by DGICYT Grants PB 93-0452 and PB 91-0307 (Spain).

Glasgow Math. J. 37 (1995) 211-219. 
polynomial $E \rightarrow c_{0}$ be weakly compact, equivalently $\hat{\Delta}^{k} E \in \mathscr{G}_{\mu}$, proving that this is the case if and only if $\mathscr{P}\left({ }^{k} E\right)$, and hence $\hat{\Delta}^{k} E$, is reflexive. These "polynomially reflexive" Banach spaces have been investigated by various authors $[1,7,9,13]$.

We also show that the situation is different if we consider the Grothendieck property of $E \hat{\otimes} F$, proving that for $E \in \mathscr{G}_{r}$ and $F^{*}$ reflexive with the bounded compact approximation property, $E \otimes F \in \mathscr{G}_{r}$ if and only if $\mathscr{L}\left(E, F^{*}\right)=\mathscr{C}_{c}\left(E, F^{*}\right)$. In particular, $\ell_{\infty} \otimes \ell_{p} \in \mathscr{G}_{r}$ for $2<p<\infty$, and $\ell_{\infty} \otimes T^{*}=\mathscr{G}_{r}$, where $T^{*}$ is the original Tsirelson space.

Every $P \in \mathscr{P}\left({ }^{k} E\right)$ has a standard extension $\tilde{P} \in \mathscr{P}\left({ }^{k} E^{* *}\right)$ (see [6]). Thus, given $P \in \mathscr{P}\left({ }^{k} E, c_{0}\right)$, with $P x=\left(P_{n} x\right)_{n}$, we can define $\tilde{P} \in \mathscr{P}\left({ }^{k} E^{* *}, \ell_{\infty}\right)$ by $\tilde{P} z:=\left(\tilde{P}_{n} z\right)_{n}$. It is shown that $E \in \mathscr{G}_{r}$ if and only if, for every sequence $\left(P_{n}\right) \subset \mathscr{P}\left({ }^{k} E\right)$ such that $P_{n} x \rightarrow 0$ for all $x \in E$, we have $\tilde{P}_{n} z \rightarrow 0$ for every $z \in E^{* *}$. Then $E \in \mathscr{G}_{r}$ if and only if for every $P \in \mathscr{P}\left({ }^{k} E, c_{0}\right)$, we have $\tilde{P}\left(E^{* *}\right) \subseteq c_{0}$.

Several authors $[\mathbf{2 0}, \mathbf{1 3}, \mathbf{1 1}]$ have studied conditions on $E, F$ so that $\mathscr{P}\left({ }^{k} E, F\right)=$ $\mathscr{P}_{c c}\left({ }^{k} E, F\right)$. Here we investigate the equality $\mathscr{P}\left({ }^{k} E, c_{0}\right)=\mathscr{P}_{c c}\left({ }^{k} E, c_{0}\right)$, proving that it is equivalent to $\mathscr{L}\left(E, c_{0}\right)=\mathscr{C} \mathscr{C}\left(E, c_{0}\right)$. Grothendieck spaces with the Dunford-Pettis property, and Schur spaces satisfy this property. In [18], examples are given of Grothendieck spaces with the Dunford-Pettis property, and semigroups of operators on these spaces are studied.

2. We first characterize the spaces $E$ such that $\mathscr{P}\left({ }^{k} E, c_{0}\right)=\mathscr{P}_{w c o}\left({ }^{k} E, c_{0}\right)$. Some previous results are needed.

Proposition 1. Let E be a nonreflexive space with the Grothendieck property. Then $E$ contains a copy of $\ell_{1}$.

Proof. Since $E \in \mathscr{G}_{k}, E$ has no quotient isomorphic to $c_{0}$. Assume $E$ contains no copy of $\ell_{1}$. Then, $E^{*}$ contains no copy of $\ell_{1}$ [12, Corollary 2.3]. Given a bounded sequence $\left(\phi_{n}\right) \subset E^{*}$, we can find a weak Cauchy subsequence $\left(\phi_{n_{k}}\right)$. The sequence $\left(\phi_{n_{k}}\right)$ is $w^{*}$-convergent, hence weakly convergent, and we conclude that $E$ is reflexive.

Proposition 2. If $\mathscr{P}\left({ }^{k} E, c_{0}\right)=\mathscr{P}_{\text {w'co }}\left({ }^{k} E, c_{0}\right)$ for some $k>1$, then $E$ is reflexive.

Proof. Suppose there is a nonweakly compact $T \in \mathscr{L}\left(E, c_{0}\right)$. Then we can find a bounded sequence $\left(x_{n}\right) \subset E$ such that $\left(T x_{n}\right)$ converges in the topology $\sigma\left(\ell_{\infty}, \ell_{1}\right)$ to some $\left(a_{n}\right) \in \ell_{\infty}$ with limsup $a_{n}=a \neq 0$. Define $P(x):=(T x)^{k}$, i.e., take the $k$ th power coordinatewise. We have $P \in \mathscr{P}_{\text {wco }}\left({ }^{k} E, c_{0}\right)$. However, $\left(P x_{n}\right)$ converges in the topology $\sigma\left(\ell_{\infty}, \ell_{1}\right)$ to the sequence $\left(a_{n}^{k}\right)_{n} \in \ell_{\infty}$, with $\limsup _{n} a_{n}^{k}=a^{k} \neq 0$, a contradiction. Hence, $E \in G_{r}$.

Suppose $E$ is nonreffexive. By Proposition $1, E$ contains a copy of $\ell_{1}$. Then, there is a quotient map $q: E \rightarrow \ell_{2}$ (see e.g. [9, Lemma 12]). Let $P: \ell_{2} \rightarrow \ell_{1}$ be the polynomial given by $P\left(\left(x_{n}\right)_{n}\right)=\left(x_{n}^{k}\right)_{n}$, and let $q^{\prime}: \ell_{1} \rightarrow c_{0}$ be a quotient map. Then the product $q^{\prime} P q \in$ $\mathscr{P}\left({ }^{k} E, c_{0}\right)$ is not weakly compact, a contradiction.

LEMMA 3. If $M$ is a complemented subspace of $E$, then $\hat{\Delta}^{k} M$ is a complemented subspace of $\hat{\Delta}^{k} E$.

Proof. If $S \in \mathscr{L}(E, E)$ is a projection with $S(E)=M$, consider the linear mapping defined by

$$
x \otimes \stackrel{(k)}{\ldots} \otimes x \mapsto S x \otimes \stackrel{(k)}{\ldots} \otimes S x \quad(x \in E)
$$


Easily, this mapping extends to an operator in $\mathscr{L}\left(\hat{\Delta}^{k} E, \hat{\Delta}^{k} E\right)$, which is the required projection.

To simplify notation, we write $x^{(k)}:=x \otimes^{(k)} \otimes x$, for $x \in E$. Let $P_{k}: \otimes^{k} E \rightarrow \otimes^{k} E$ be the projection defined by

$$
P_{k}\left(x_{1} \otimes \ldots \otimes x_{k}\right)=\frac{1}{k ! 2^{k}} \sum_{\epsilon_{j}= \pm 1} \epsilon_{1} \ldots \epsilon_{k}\left(\epsilon_{1} x_{1}+\ldots+\epsilon_{k} x_{k}\right)^{(k)} .
$$

This mapping extends to an operator on $\hat{\otimes}^{k} E$, which is a projection of $\hat{\otimes}^{k} E$ onto $\hat{\Delta}^{k} E$. The following Lemma is contained in [8]. We include the proof for completeness.

LEMMA 4. Given $u \in \hat{\Delta}^{k} E$, there exists a sequence $\left(x_{i}\right) \subset E$ such that $\sum_{i=1}^{\infty}\left\|x_{i}\right\|^{k}<\infty$ and $u=\sum_{i=1}^{\infty} \epsilon_{i} x_{i}^{(k)}$, with $\epsilon_{i} \in\{ \pm 1\}$.

Proof. By the definition of the projective norm, for any $\delta>0$, we can find sequences $\left(x_{n}^{1}\right), \ldots,\left(x_{n}^{k}\right) \subset E$, such that

$$
\sum_{n=1}^{\infty}\left\|x_{n}^{1}\right\| \cdot \ldots \cdot\left\|x_{n}^{k}\right\|<\|u\|+\delta, \quad \text { and } \quad u=\sum_{n=1}^{\infty} x_{n}^{1} \otimes \ldots \otimes x_{n}^{k}
$$

Then,

$$
u=P_{k} u=\sum_{n=1}^{\infty} P_{k}\left(x_{n}^{1} \otimes \ldots \otimes x_{n}^{k}\right)
$$

We can assume that, for each $n,\left\|x_{n}^{1}\right\|=\ldots=\left\|x_{n}^{k}\right\|$. Since

$$
P_{k}\left(x_{n}^{1} \otimes \ldots \otimes x_{n}^{k}\right)=\frac{1}{k ! 2^{k}} \sum_{\epsilon_{j}= \pm 1} \epsilon_{1} \ldots \epsilon_{k}\left(\epsilon_{1} x_{n}^{1}+\ldots+\epsilon_{k} x_{n}^{k}\right)^{(k)},
$$

denoting by $\left(x_{i}\right)$ the sequence

$$
\left\{\frac{1}{2 \sqrt[k]{k !}}\left(\epsilon_{1} x_{n}^{1}+\ldots+\epsilon_{k} x_{n}^{k}\right): \epsilon_{1}, \ldots, \epsilon_{k}= \pm 1 ; n=1,2, \ldots\right\},
$$

we obtain

$$
\sum_{i=1}^{\infty}\left\|x_{i}\right\|^{k} \leq \frac{k^{k}}{k !}(\|u\|+\delta), \quad \text { and } \quad u=\sum_{i=1}^{\infty} \epsilon_{i} x_{i}^{(k)},
$$

completing the proof.

Before stating the main result of this part, recall that for $P \in \mathscr{P}\left({ }^{k} E, F\right)$, its adjoint is the operator $P^{*}: F^{*} \rightarrow \mathscr{P}\left({ }^{k} E\right)$ given by $P^{*}(\psi)=\psi \circ P$ for every $\psi \in F^{*}$. Then $P \in$ $\mathscr{P}_{\text {wco }}\left({ }^{k} E, F\right)$ if and only if $P^{*}$ is weakly compact [23, Proposition 2.1].

Theorem 5. Given $k>1$, we have that $\mathscr{P}\left({ }^{k} E, c_{0}\right)=\mathscr{P}_{\text {wco }}\left({ }^{k} E, c_{0}\right)$ if and only if the space $\mathscr{P}\left({ }^{k} E\right)$ is reflexive.

Proof. For the "only if" part, if $E$ is separable, then $\hat{\Delta}^{k} E$ is a Grothendieck 
separable space, hence reflexive. In the general case, suppose $\hat{\Delta}^{k} E$ is not reflexive. By Lemma 4, we can find

$$
w_{n}=\sum_{i=1}^{\infty} \epsilon_{n}^{i} x_{n}^{i} \otimes \ldots \otimes x_{n}^{i}, \quad\left\|w_{n}\right\| \leq 1
$$

so that $\left\{w_{n}\right\}$ is not relatively weakly compact in $\hat{\Delta}^{k} E$. Let $N$ be the closed linear span of $\left\{x_{n}^{i}\right\}_{i, n}$ in $E$. Since $E$ is reflexive (Proposition 2), there is a separable subspace $M$ complemented in $E$ with $N \subseteq M$. By Lemma $3, \hat{\Delta}^{k} M \in \mathscr{G}$, and is separable, therefore reflexive. However, $\left(w_{n}\right) \subset \hat{\Delta}^{k} M$, a contradiction, and we conclude that $\hat{\Delta}^{k} E$ and $\mathscr{P}\left({ }^{k} E\right)$ are reflexive.

The "if" part is clear by the previous comment.

It is well known that the space $\mathscr{P}\left({ }^{k} \ell_{p}\right)$ is reflexive if and only if $k<p<\infty$. If $E=T^{*}$, then $\mathscr{P}\left({ }^{k} E\right)$ is reflexive for all $k[1]$.

In the last Theorem, $c_{0}$ can be replaced by any superspace $F$. However, for $F$ containing no copy of $c_{0}$, the result is not true since, for instance, every polynomial from $E=c_{0}$ into $F \not c_{0}$ is weakly continuous on bounded subsets (see e.g. [11]).

3. In this part, we show that the situation is different for general projective tensor products. We refine a result of [17], proving that for $E \in \mathscr{G}_{\mu}$ and $F^{*}$ reflexive with the bounded compact approximation property, $E \hat{\otimes} F \in \mathscr{G}_{*}$ if and only if $\mathscr{L}\left(E, F^{*}\right)=$ $\mathscr{C}_{0}\left(E, F^{*}\right)$. As a consequence, $\ell_{\infty} \hat{\otimes} \ell_{p}$ for $2<p<\infty$ and $\ell_{\infty} \otimes T^{*}$ have the Grothendieck property. reflexive.

Proposition 6. Suppose $E \otimes F \in \mathscr{G}_{r}$. Then $E, F \in \mathscr{G}_{r}$ and at least one of them is

Proof. Since $E$ and $F$ are complemented in $E \hat{\otimes} F$, the first assertion is clear. Suppose $E$ and $F$ are nonreflexive. Then each of them contains a copy of $\ell_{1}$ (Proposition 1). Hence, there are quotient maps (see e.g. [9, Lemma 12])

$$
q_{1}: E \rightarrow \ell_{2} \text { and } q_{2}: F \rightarrow \ell_{2}
$$

Consider the quotient maps

$$
E \hat{\otimes} F \stackrel{q_{1} \hat{\otimes} \mathrm{id}}{\longrightarrow} \ell_{2} \hat{\otimes} F \stackrel{\text { id } \hat{\otimes} q_{2}}{\longrightarrow} \ell_{2} \hat{\otimes} \ell_{2}
$$

If is well known that $\ell_{2} \hat{\otimes} \ell_{2} \notin \mathscr{G}_{r}$ (separable Grothendieck spaces are reflexive). Hence, $E \hat{\otimes} F \notin \mathscr{G}$, a contradiction.

Remark 7. It follows from Proposition 6 that whenever $E_{1} \hat{\otimes} \ldots \hat{\otimes} E_{k} \in \mathscr{G}_{k}$ and, for example, $E_{1}$ is not reflexive, $E_{2} \hat{\otimes} \ldots \hat{\otimes} E_{k}$ is reflexive. In particular, $E_{2}, \ldots, E_{k}$ are reflexive.

Before stating the next result, recall that the dual of $E \otimes F$ may be identified with $\mathscr{L}\left(E, F^{*}\right)$. 
Proposition 8. Assume $E \in \mathscr{G}_{k}$ and $F$ is reflexive. If $\mathscr{L}\left(E, F^{*}\right)=\mathscr{C}_{c}\left(E, F^{*}\right)$, then $E \hat{\otimes} F \in \mathscr{G}$.

Proof. Let $\left(A_{n}\right) \subset \mathscr{L}\left(E, F^{*}\right)$ be a $w^{*}$-null sequence. Then for every $x \in E$ and $y \in F$,

$$
\left\langle y, A_{n}(x)\right\rangle=\left\langle x \otimes y, A_{n}\right\rangle \rightarrow 0 .
$$

Applying Kalton's test for the weak convergence of sequences in spaces of compact operators (see Theorem 3 in [16]), we have that $\left(A_{n}\right)$ is weakly null.

We say that $E$ has the bounded compact approximation property (BCAP) [4] if there exists $\lambda \geq 1$ so that for each compact subset $K \subset E$ and for each $\epsilon>0$ there is $S \in \mathscr{C}_{c}(E, E)$ such that

$$
\sup \{\|S x-x\|: x \in K\} \leq \epsilon, \quad\|S-\mathrm{id}\| \leq \lambda .
$$

Every space with the bounded approximation property has the BCAP. The converse is not true [24].

Proposition 9. Suppose $F^{*}$ is reflexive and has the BCAP, and $E \hat{\otimes} F \in \mathscr{G}_{\text {r. Then }}$ we have $\mathscr{L}\left(E, F^{*}\right)=\mathscr{C}_{r}\left(E, F^{*}\right)$.

Proof. Suppose first that $F^{*}$ is separable. Then there is a bounded sequence $\left(T_{n}\right) \subset \mathscr{C}_{n}\left(F^{*}, F^{*}\right)$ such that $T_{n} \psi \rightarrow \psi$ for all $\psi \in F^{*}$. Assume $T \in \mathscr{L}\left(E, F^{*}\right)$ is not compact. For $x \in E, y \in F$ we have

$$
\left\langle x \otimes y, T_{n} T\right\rangle=\left\langle y, T_{n}(T x)\right\rangle \rightarrow\langle y, T x\rangle=\langle x \otimes y, T\rangle .
$$

Since $\left(T_{n} T\right)$ is bounded and $\{x \otimes y: x \in E, y \in F\}$ generates a dense subset of $E \hat{\otimes} F$, we have that $\left(T_{n} T\right)$ is $w^{*}$-convergent to $T$. Since $\left(T_{n} T\right) \subset \mathscr{C}_{c}\left(E, F^{*}\right),\left(T_{n} T\right)$ is not weakly convergent to $T$, a contradiction.

For $F^{*}$ nonseparable, suppose $T$ as above. There is a bounded sequence $\left(x_{n}\right) \subset E$ such that $\left(T x_{n}\right)$ has no Cauchy subsequence. The closed linear span of $\left\{T x_{n}\right\}$ is contained in a separable space $M^{*}$ complemented in $F^{*}$. If $q: F^{*} \rightarrow M^{*}$ is the identity on $M^{*}$, then $q T \in \mathscr{L}\left(E, M^{*}\right)$ is noncompact. By the above, $E \hat{\otimes} M \notin \mathscr{G}_{\mu}$, a contradiction since $E \hat{\otimes} M$ is a quotient of $E \otimes F$.

COROLlary 10 . For $1 \leq p \leq \infty$, the space $\ell_{\infty} \otimes \ell_{p}$ has the Grothendieck property if and only if $2<p<\infty$.

Proof. If $2<p<\infty$, we have $\ell_{p}^{*}=\ell_{q}$ with $1<q<2$, and it is known that every operator $\ell_{\infty} \rightarrow \ell_{q}$ factors through $\ell_{2}$ [21, Corollary 4.4] and is therefore compact. The converse is easy.

REMARK 11. (a) We note that the space $\ell_{\infty} \otimes \ell_{p} \otimes \ell_{p}$ does not have the Grothendieck property, for $2 \leq p \leq 3$. Indeed, there is a noncompact operator $T: \ell_{p} \rightarrow\left(\ell_{p} \otimes \ell_{p}\right)^{*}$, for instance, the operator $T$ associated to the polynomial $P x:=\sum_{i=1}^{\infty} x_{i}^{3}$, for $x=\left(x_{i}\right) \in \ell_{p}$, given by $(T x)(y \otimes z)=\hat{P}(x, y, z)$, where $\hat{P}$ is the symmetric 3 -linear form associated to $P$. Then 
$T$ is not completely continuous, so we can find a weakly null sequence $\left(x_{n}\right) \subset \ell_{p}$ such that $\left\{T x_{n}\right\}$ is not relatively compact. Passing to a subsequence, we can assume that $\left(x_{n}\right)$ is equivalent to a block basis and hence equivalent to the basis of $\ell_{p}$. Let $q: \ell_{\infty} \rightarrow \ell_{2}$ be a quotient, and $j: \ell_{2} \rightarrow \ell_{p}$ the operator taking the $\ell_{2}$-basis into $\left(x_{n}\right)$. Then Tjq: $\ell_{\infty} \rightarrow$ $\left(\ell_{p} \otimes \ell_{p}\right) *$ is not compact, and it is enough to apply Proposition 9.

(b) It is proved in $[2$, Corollary 8$]$ that the space $\left(\hat{\otimes}^{k} T^{*}\right) \hat{\otimes} \ell_{p}$ is reflexive, for $1<p<\infty$.

\section{COROLlaRy 12. The space $\ell_{\infty} \hat{\otimes} T^{*}$ has the Grothendieck property.}

The proof relies on the following Lemma.

Lemma 13. Let $T$ be the dual of $T^{*}$. Then $\mathscr{L}\left(\ell_{\infty}, T\right)=\mathscr{C}_{c}\left(\ell_{\infty}, T\right)$.

Proof. Assume $S \in \mathscr{L}\left(\ell_{\infty}, T\right)$ is not compact. Let $\left(y_{k}\right) \subset \ell_{\infty}$ be a bounded sequence such that $\left(S y_{k}\right)$ has no convergent subsequence. Choose a weakly convergent subsequence $\left(S y_{k_{n}}\right)$, and take $x_{n}:=y_{k_{2 n}}-y_{k_{2 n-1}}$. Then $\left(S x_{n}\right)$ is weakly null, and we can assume that it is equivalent to a block basis in $T$.

Since $\left\{S x_{n}\right\}$ spans a complemented subspace [S $\left.x_{n}\right]$ [5, Proposition II.6], there is an operator $V: T \rightarrow\left[S x_{n}\right]$ which is the identity on $\left[S x_{n}\right]$. For $1<q<2, T$ has lower $q$-estimates [5, Proposition V.10], so there is an operator $U:\left[S x_{n}\right] \rightarrow \ell_{q}$ given by $U\left(S x_{n}\right)=e_{n}$, where $\left(e_{n}\right)$ is the unit vector basis of $\ell_{q}$. Then $U V S: \ell_{x} \rightarrow \ell_{q}$ is not compact, a contradiction [21, Corollary 4.4].

In [17], the following result was obtained (see Zentralblatt Math. 599 \#6017 (1987)):

"Let $E$ be a Banach space with the Grothendieck property, and $F$ a reflexive space with the metric approximation property. For $E \hat{\otimes} F$ to have the Grothendieck property it is necessary and sufficient, that each operator $E \rightarrow F^{*}$ be compact".

Another related result is the following of [15]:

"If $E$ and $F$ are reflexive and both have the approximation property, then $\mathscr{L}(E, F)$ is reflexive if and only if $\mathscr{L}(E, F)=\mathscr{C}_{0}(E, F)$ ".

4. Next we describe the Grothendieck property in terms of polynomials. Recall that each $P \in \mathscr{P}\left({ }^{k} E\right)$ has a Davie-Gamelin extension $\tilde{P} \in \mathscr{P}\left({ }^{k} E^{* *}\right)$ (see the Introduction). The authors are indebted to Professor Richard M. Aron, who suggested this study. Namely, he asked if, given $E \in \mathscr{G}_{\mu}$ and a sequence $\left(P_{n}\right) \subset \mathscr{P}\left({ }^{k} E\right)$ with $P_{n} x \rightarrow 0$ for all $x \in E$, it is true that $\tilde{P}_{n} z \rightarrow 0$ for all $z \in E^{* *}$. The following theorem shows that the answer is affirmative.

THEOREM 14. The following assertions are equivalent:

(a) E has the Grothendieck property;

(b) for every integer $k$, given a sequence $\left(P_{n}\right) \subset \mathscr{P}\left({ }^{k} E\right)$ with $P_{n} x \rightarrow 0$ for all $x \in E$, then $\tilde{P}_{n} z \rightarrow 0$ for all $z \in E^{* *}$;

(c) the same statement as (b) is true for some $k$.

Proof. (a) $\Rightarrow$ (b). By induction on $k$. For $k=1$, the result is nothing but the definition of the Grothendieck property. Suppose it holds for $k-1$, and let $\left(P_{n}\right) \subset \mathscr{P}\left({ }^{k} E\right)$ be a sequence such that $P_{n} x \rightarrow 0$ for all $x \in E$. Denote by $F_{n} \in \mathscr{L}\left({ }^{k} E\right)$ the associated symmetric $k$-linear form, and by $G_{n} \in \mathscr{L}\left(E \times E^{* *} \times{ }^{(k-1)} \times E^{* *}\right)$ an extension obtained by the Davie-Gamelin method. 
Thanks to the polarization formula [19, Theorem 1.10], we have that

$$
F_{n}\left(x_{1}, x_{2}, \ldots, x_{k}\right) \rightarrow 0 \text { for every } x_{1}, \ldots, x_{k} \in E .
$$

Fixing $x_{1} \in E$, we define $Q_{n} \in \mathscr{P}\left({ }^{k-1} E\right)$ by $Q_{n}(x)=F_{n}\left(x_{1}, x, \ldots, x\right)$. Then $Q_{n} x \rightarrow 0$ for all $x \in E$.

By the induction hypothesis and polarization,

$$
G_{n}\left(x_{1}, z_{2}, \ldots, z_{k}\right) \rightarrow 0, \text { for } z_{2}, \ldots, z_{k} \in E^{* *} .
$$

Then, for $z_{2}, \ldots, z_{k} \in E^{* *}$ fixed, the sequence $\left(\phi_{n}\right) \subset E^{*}$, given by $\phi_{n}(x)=$ $G_{n}\left(x, z_{2}, \ldots, z_{k}\right)$, is $w^{*}$-null, hence weakly null, and so

$$
\tilde{F}_{n}\left(z_{1}, z_{2}, \ldots, z_{k}\right) \rightarrow 0 \text { for every } z_{1}, \ldots, z_{k} \in E^{* *},
$$

where $\tilde{F}_{n} \in \mathscr{L}\left({ }^{k} E^{* *}\right)$ is the Davie-Gamelin extension of $F_{n}$.

(b) $\Rightarrow$ (c) is trivial.

(c) $\Rightarrow$ (a). Given a $w^{*}$-null sequence $\left(\phi_{n}\right) \subset E^{*}$, apply (c) to $P_{n} x:=\left(\phi_{n}(x)\right)^{k}$.

Given a polynomial $P \in \mathscr{P}\left({ }^{k} E, c_{0}\right)$, with $P x=\left(P_{n} x\right)_{n}$, we define $\tilde{P} \in \mathscr{P}\left({ }^{k} E^{* *}, \ell_{\infty}\right)$ by $\tilde{P} z:=\left(\tilde{P}_{n} z\right)_{n}$. Then we have the following corollary.

COROLlaRY 15. The space $E$ has the Grothendieck property if and only if for every $P \in \mathscr{P}\left({ }^{k} E, c_{0}\right)$, we have that $\tilde{P}\left(E^{* *}\right) \subseteq c_{0}$.

For polynomials whose restrictions to bounded sets are weakly continuous, we can deduce a result on weak convergence.

COROLlaRy 16. The following assertions are equivalent:

(a) E has the Grothendieck property;

(b) for every integer $k$ and every $F$, if for a sequence $\left(P_{n}\right) \subset P_{w b}\left({ }^{k} E, F\right)$ we have that $\left\langle P_{n} x, \psi\right\rangle \rightarrow 0$ for all $x \in E$ and $\psi \in F^{*}$, then $\left(P_{n}\right)$ is weakly null;

(c) the same statement as (b) is true for some $k$ and some $F \neq\{0\}$;

(d) for some $k$, if for a sequence $\left(P_{n}\right) \subset \mathscr{P}_{w b}\left({ }^{k} E\right)$ we have that $P_{n} x \rightarrow 0$ for all $x \in E$, then $\left(P_{n}\right)$ is weakly null.

Proof. (a) $\Rightarrow$ (b). It is proved in Theorem 4 of $[\mathbf{1 0}]$ that a sequence $\left(P_{n}\right) \subset \mathscr{P}_{w b}\left({ }^{k} E, F\right)$ is weakly null if and only if, for every $z \in E^{* *}$ and $\psi \in F^{*}$, we have $\left\langle\tilde{P}_{n} z, \psi\right\rangle \rightarrow 0$. Therefore, it is enough to apply Theorem 14(b).

(b) $\Rightarrow$ (c) is trivial.

(c) $\Rightarrow(\mathrm{d})$. Take $0 \neq y \in F$ and define $Q_{n} x:=\left(P_{n} x\right) y$.

(d) $\Rightarrow$ (a). Given a $w^{*}$-null sequence $\left(\phi_{n}\right) \subset E^{*}$, apply (d) to $P_{n} x:=\left(\phi_{n}(x)\right)^{k}$.

5. Several authors $[\mathbf{2 0}, \mathbf{1 3}, \mathbf{1 1}]$ have studied conditions on $E, F$ so that $\mathscr{P}\left({ }^{k} E, F\right)=$ $\mathscr{P}_{c c}\left({ }^{k} E, F\right)$. Here we investigated the equality $\mathscr{P}\left({ }^{k} E, c_{0}\right)=\mathscr{P}_{c c}\left({ }^{k} E, c_{0}\right)$, proving that it is equivalent to $\mathscr{L}\left(E, c_{0}\right)=\mathscr{C} \mathscr{C}\left(E, c_{0}\right)$. Therefore, the Grothendieck spaces with the Dunford-Pettis property, and the Schur spaces satisfy this property.

THEOREM 17. The following assertions are equivalent:

(a) $\mathscr{L}\left(E, c_{0}\right)=\mathscr{C} \mathscr{C}\left(E, c_{0}\right)$

(b) $\mathscr{P}\left({ }^{k} E, c_{0}\right)=\mathscr{P}_{c c}\left({ }^{k} E, c_{0}\right)$ for all integers $k$.

(c) $\mathscr{P}\left({ }^{k} E, c_{0}\right)=\mathscr{P}_{c c}\left({ }^{k} E, c_{0}\right)$ for some integer $k$. 
Proof. (a) $\Rightarrow$ (b). By induction on $k$. For $k=1$ there is nothing to prove. Assume the result is true for $k-1$, and consider $P \in \mathscr{P}\left({ }^{k} E, c_{0}\right)$ with associated $k$-linear mapping $A$. We only sketch the proof, since it follows the lines of that in [11, Theorem 6]. In fact, we can prove that every $k$-linear mapping from $E^{k}$ into $c_{0}$ takes weak Cauchy sequences into convergent ones. Let $\left(x_{n}^{1}\right), \ldots,\left(x_{n}^{k}\right) \subset E$ be weak Cauchy sequences. Suppose first that one of them, say $\left(x_{n}^{1}\right)$, is weakly null.

Define the operator $T: E \rightarrow c_{0}\left(c_{0}\right)$ by

$$
y \mapsto\left(A\left(x_{n}^{1}, \ldots, x_{n}^{k-1}, y\right)\right)_{n} .
$$

Using the induction hypothesis, it is not difficult to see that $T$ is well-defined. Since $c_{0}\left(c_{0}\right)$ is isomorphic to $c_{0}, T$ is completely continuous. From this, we have

$$
\lim _{m}\left\|A\left(x_{m}^{1}, \ldots, x_{m}^{k}\right)\right\| \leq \lim _{m} \sup _{n}\left\|A\left(x_{n}^{1}, \ldots, x_{n}^{k-1}, x_{m}^{k}\right)\right\|=0 .
$$

In the general case, the proof follows that of Theorem 6 in [11].

(b) $\Rightarrow$ (c) is obvious.

(c) $\Rightarrow$ (a) is clear.

The condition $\mathscr{L}\left(E, c_{0}\right)=\mathscr{C} \mathscr{C}\left(E, c_{0}\right)$ implies that $E$ has the Dunford-Pettis property. However, there are spaces with the Dunford-Pettis property that admit non-completely continuous operators into $c_{0}$ (e.g. $E=c_{0}, E=L_{1}[0,1]$ ).

Added in Proof. While this paper was in press, G. Emmanuele pointed out that Propositions 6 and 8 and Corollary 10 are contained in his note About certain isomorphic properties of Banach spaces in projective tensor products, Extracta Math. 5 (1990), 23-25.

\section{REFERENCES}

1. R. Alencar, R. M. Aron and S. Dineen, A reflexive space of holomorphic functions in infinitely many variables, Proc. Amer. Math. Soc. 90 (1984), 407-411.

2. R. Alencar, R. M. Aron and G. Fricke, Tensor products of Tsirelson's space, Illinois J. Math. 31 (1987), 17-23.

3. R. M. Aron, C. Hervés and M. Valdivia, Weakly continuous mappings on Banach spaces, J. Funct. Anal. 52 (1983), 189-204.

4. K. Astala and H. O. Tylli, On the bounded compact approximation property and measures of noncompactness, J. Funct. Anal. 70 (1987), 388-401.

5. P. G. Casazza and T. J. Shura, Tsirelson's Space, Lecture Notes in Math. 1363 (Springer-Verlag 1989).

6. A. M. Davie and T. W. Gamelin, A theorem on polynomial-star approximation, Proc. Amer. Math. Soc. 106 (1989), 351-356.

7. J. D. Farmer, Polynomial reflexivity in Banach spaces, Israel J. Math. 87 (1994), 257-273.

8. M. González, Remarks on $Q$-reflexive Banach spaces, preprint.

9. M. González and J. M. Gutiérrez, Unconditionally converging polynomials on Banach spaces, Math. Proc. Cambridge Philos. Soc., to appear.

10. M. González and J. M. Gutiérrez, Weak compactness in spaces of differentiable mappings, Rocky Mountain J. Math., to appear.

11. M. González and J. M. Gutiérrez, When every polynomial is unconditionally converging, Arch. Math. 63 (1994), 145-151.

12. M. González and V. Onieva, Lifting results for sequences in Banach spaces, Math. Proc. Cambridge Philos. Soc. 105 (1989), 117-121. 
13. R. Gonzalo and J. A. Jaramillo, Compact polynomials between Banach spaces, preprint.

14. J. M. Gutiérrez, Weakly continuous functions on Banach spaces not containing $\ell_{1}$, Proc. Amer. Math. Soc. 119 (1993), 147-152.

15. J. R. Holub, Reflexivity of $\mathscr{L}(E, F)$, Proc. Amer. Math. Soc. 39 (1973), 175-177.

16. N. J. Kalton, Spaces of compact operators, Math. Ann. 208 (1974), 267-278.

17. V. Khasanov, On Banach spaces with Grothendieck property (Russian), in Extremal problems of the theory of functions, Collect. Articles, Tomsk 1984, 85-96.

18. H. P. Lotz, Uniform convergence of operators on $L_{\alpha}$ and similar spaces, Math. Z. 190 (1985), 207-220.

19. J. Mujica, Complex Analysis in Banach Spaces, Math. Studies 120 (North-Holland 1986).

20. A. Pelczyński, A property of multilinear operations, Studia Math. 16 (1957), 173-182.

21. G. Pisier, Factorization of Linear Operators and Geometry of Banach Spaces, Reg. Conf. Ser. Math. 60 (American Mathematical Society 1986).

22. R. A. Ryan, Applications of topological tensor products to infinite dimensional holomorphy, Ph.D. thesis, Trinity College, Dublin 1980.

23. R. A. Ryan, Weakly compact holomorphic mappings on Banach spaces, Pacific J. Math. 131 (1988), 179-190.

24. G. Willis, The compact approximation property does not imply the approximation property, Studia Math. 103 (1992), 99-108.

Departamento de Matemáticas

Facultad de Ciencias

UNIVERSIDAD DE CANTABRIA

39071 SANTANDER

SPAIN
Departamento de Matemática Aplicada

ETS DE INGENIEROS INDUSTRIALES

UNIVERSIDAD PolitéCNICA DE MADRID

C. José Gutiérrez Abascal 2

28006 MADRID

SPAIN 